\title{
Exponential mean-square stability properties of stochastic linear multistep methods
}

\author{
Evelyn Buckwar ${ }^{1} \cdot$ Raffaele D'Ambrosio ${ }^{2}$
}

Received: 12 November 2019 / Accepted: 17 June 2021 / Published online: 26 July 2021

(C) The Author(s) 2021

\begin{abstract}
The aim of this paper is the analysis of exponential mean-square stability properties of nonlinear stochastic linear multistep methods. In particular it is known that, under certain hypothesis on the drift and diffusion terms of the equation, exponential meansquare contractivity is visible: the qualitative feature of the exact problem is here analysed under the numerical perspective, to understand whether a stochastic linear multistep method can provide an analogous behaviour and which restrictions on the employed stepsize should be imposed in order to reproduce the contractive behaviour. Numerical experiments confirming the theoretical analysis are also given.
\end{abstract}

Keywords Exponential mean-square stability $\cdot$ Exponential mean-square contractivity $\cdot$ Nonlinear stochastic differential equations $\cdot$ Stochastic linear multistep methods $\cdot$ Nonlinear stability

Mathematics Subject Classification (2010) 60H35 · 65C30

\section{Framework}

The purpose of this paper is the analysis of nonlinear stability properties of stochastic numerical methods, in the spirit of preserving along the numerical solution a qualitative behaviour that is apparent in the solution of the continuous problem. Once

Communicated by: Ivan Oseledets

Raffaele D'Ambrosio

rdambrosio@unisa.it

Evelyn Buckwar

evelyn.buckwar@jku.at

1 Institut für Stochastik, Johannes Kepler University of Linz, Altenbergerstrasse 69, 4040,

Linz, Austria

2 Department of Mathematics, University of Salerno, via Giovanni Paolo II, 132 - 84084, Fisciano (Sa), Italy 
the property of the continuous problem is detected and identified through sufficient conditions, we aim to provide their numerical counterparts, equivalent to conditional stability properties for the numerical method. Before entering into the details, we first recover here the deterministic case, with special emphasis on linear and nonlinear stability properties.

\subsection{Linear and nonlinear stability issues for deterministic differential equations}

For a well-posed deterministic initial value problem based on the ordinary differential equation

$$
y^{\prime}(t)=f(y(t))
$$

linear stability properties are classically provided with respect to the scalar linear test equation

$$
y^{\prime}(t)=\xi y(t), \quad t \geq 0,
$$

where $\xi \in \mathbb{C}$ and $\operatorname{Re}(\xi) \leq 0$, considered for the first time by Dahlquist [6]. The solution of this simple problem remains bounded when $t$ goes to infinity and one needs to require that the numerical solution possesses an analogous stability property to that displayed by the exact solution: this fact is at the basis of the linear stability analysis of numerical methods (see [4] and references therein).

Based on the concept of one-sided Lipschitz continuity G. Dahlquist laid the foundation of the theory of nonlinear stability in [7] (this paper has also been celebrated by J. Butcher in the review paper [3]), in order to treat situations beyond the realm of linear stability analysis. Hence, the following nonlinear test problem

$$
\left\{\begin{array}{l}
y^{\prime}(t)=g(t, y(t)), t \geq 0, \\
y(0)=y_{0}
\end{array}\right.
$$

is considered, with $g: \mathbb{R} \times \mathbb{R}^{m} \rightarrow \mathbb{R}^{m}$ satisfying a one-sided Lipschitz condition of the form

$$
\left(g\left(t, y_{1}\right)-g\left(t, y_{2}\right)\right)^{\top}\left(y_{1}-y_{2}\right) \leq 0
$$

for all $t \geq 0$ and $y_{1}, y_{2} \in \mathbb{R}^{m}$. Denote by $y(t)$ and $\tilde{y}(t)$ two solutions to (2) with initial conditions $y_{0}$ and $\tilde{y}_{0}$, respectively. Then it is known (see $[3,9,11]$ and references therein) that the condition (3) implies the contractivity of the trajectories, given by the inequality

$$
\left\|y\left(t_{2}\right)-\tilde{y}\left(t_{2}\right)\right\| \leq\left\|y\left(t_{1}\right)-\tilde{y}\left(t_{1}\right)\right\|
$$

for $0 \leq t_{1} \leq t_{2}$, where $\|\cdot\|$ is any norm in $\mathbb{R}^{m}$, and the corresponding problem is said to be dissipative.

Extensive research has been carried out in order to provide numerical methods generating contractive numerical solutions for dissipative problems, giving rise to the notions of AN-stability, G-stability, algebraic stability and so on (see $[4,15]$ and references therein).

\subsection{Exponential mean-square stability of stochastic differential equations}

We now move to the main issue of this paper, i.e. a nonlinear Itô stochastic differential equation (SDE)

$$
d X(t)=f(X(t)) d t+g(X(t)) d W(t)
$$


for $t \in[0, T]$, where $f: \mathbb{R}^{n} \rightarrow \mathbb{R}^{n}, g: \mathbb{R}^{n} \rightarrow \mathbb{R}^{n \times m}$, and $W(t)$ is an $m$-dimensional Wiener process.

Following [12], we assume the following conditions on the functions $f$ and $g$ :

(i) $f, g \in C^{1}$;

(ii) the drift coefficient $f$ satisfies a one-sided Lipschitz condition, i.e. there exists $\mu \in \mathbb{R}$ such that

$$
\langle x-y, f(x)-f(y)\rangle \leq \mu|x-y|^{2}, \quad \forall x, y \in \mathbb{R}^{n} ;
$$

(iii) the diffusion coefficient $g$ is globally Lipschitz, i.e. there exists $L>0$ such that

$$
|g(x)-g(y)|^{2} \leq L|x-y|^{2}, \quad \forall x, y \in \mathbb{R}^{n} .
$$

Under the aforementioned assumptions, the following result holds [12, 14].

Theorem 1 Under conditions (i)-(iii), any two solutions $X(t)$ and $Y(t)$ of (5) with $\mathbb{E}\left|X_{0}\right|^{2}<\infty$ and $\mathbb{E}\left|Y_{0}\right|^{2}<\infty$ satisfy

$$
\mathbb{E}|X(t)-Y(t)|^{2} \leq \mathbb{E}\left|X_{0}-Y_{0}\right|^{2} e^{\alpha t},
$$

where

$$
\alpha=2 \mu+L
$$

As a consequence of this result, the following definition is provided [12].

Definition 1 The solution of any given SDE (5) satisfying (8) with $\alpha<0$ is said to be exponential mean-square contractive.

For other possible stability issues, compare for instance $[1,10,18]$ and references therein.

We aim to investigate the numerical counterpart of (8) when trajectories are generated by stochastic linear multistep methods, in order to provide bounds on the stepsize whose values eventually reproduce such a contractive behaviour, according to the following definition.

Definition 2 Assuming that the integration interval $[0, T]$ of $(5)$ is discretized in the following grid

$$
\left\{t_{n}=n \Delta t, n=0,1, \ldots, N, \Delta t=T / N\right\},
$$

the numerical solution of any given $\operatorname{SDE}$ (5) satisfying (8) with $\alpha<0$ is said to be exponential mean-square contractive if there exists $v<0$ such that

$$
\mathbb{E}\left|X_{n}-Y_{n}\right|^{2} \leq \mathbb{E}\left|X_{0}-Y_{0}\right|^{2} e^{v t_{n}}, \quad n=1,2, \ldots, N
$$

In other terms, (9) provides the discrete time counterpart of (8). The article proceeds as follows: Section 2 provides inequalities of type (9) when linear multistep methods (10) are used; Sections 3 and 4 are respectively devoted to the analysis of the exponential mean-square contractive behaviour of one and two-step methods; Section 5 contains the numerical results obtained by applying the analysed methods 
to scalar nonlinear equations as well as to systems of equations; Section 6 is devoted to giving some concluding remarks.

\section{Exponential mean-square stability analysis}

We develop a general analysis of the exponential mean-square stability properties of the general family of stochastic two-step methods [2]

$$
\begin{aligned}
\alpha_{2} X_{n+1}+\alpha_{1} X_{n}+\alpha_{0} X_{n-1}= & \Delta t\left(\beta_{2} f\left(X_{n+1}\right)+\beta_{1} f\left(X_{i}\right)+\beta_{0} f\left(X_{n-1}\right)\right) \\
& +\gamma_{1} g\left(X_{n}\right) \Delta W_{n}+\gamma_{0} g\left(X_{n-1}\right) \Delta W_{n-1} .
\end{aligned}
$$

In our analysis, the following lemma, reproduced from [12, 13], will be particularly useful.

Lemma 1 Under conditions (6) and (7), given $b_{1}, b_{2} \in \mathbb{R}^{n}$ and $h \in(0,1 /(2 \beta))$, with $\beta=\max \left(\mu+\frac{1}{2}, L\right)$, let $a_{1}, a_{2} \in \mathbb{R}^{n}$ satisfy the implicit equation

$$
a_{i}-h f\left(a_{i}\right)=b_{i}, \quad i=1,2 .
$$

Then, $a_{1}$ and $a_{2}$ exist, are unique and satisfy the inequality

$$
(1-2 h \mu)\left|a_{1}-a_{2}\right|^{2} \leq\left|b_{1}-b_{2}\right|^{2} .
$$

For two-step methods (10), the following three-terms inequality holds.

Theorem 2 Under conditions (i)-(iii), any two numerical solutions $\left\{X_{n}\right\}_{n}$ and $\left\{Y_{n}\right\}_{n}$ with initial values such that $\mathbb{E}\left|X_{0}\right|^{2}<\infty$ and $\mathbb{E}\left|Y_{0}\right|^{2}<\infty$, generated by applying a two-step method (10) with stepsize $\Delta t$ satisfy

$$
\mathbb{E}\left|X_{n+1}-Y_{n+1}\right|^{2} \leq \beta_{\mathrm{TS}} \mathbb{E}\left|X_{n}-Y_{n}\right|^{2}+\gamma_{\mathrm{TS}} \mathbb{E}\left|X_{n-1}-Y_{n-1}\right|^{2},
$$

where $\beta_{T S}$ and $\gamma_{T S}$ are the expressions

$$
\begin{aligned}
& \beta_{\mathrm{TS}}=\frac{\alpha_{1}^{2}+\alpha_{0} \alpha_{1}-\left(2 \alpha_{1} \beta_{1} \mu+\left(\alpha_{1} \beta_{0}+\alpha_{0} \beta_{1} M\right)-\gamma_{1}^{2} L\right) \Delta t+\left(\beta_{1}^{2}+\beta_{1} \beta_{0}\right) M \Delta t^{2}}{\alpha_{2}^{2}\left(1-2 \beta_{2} \mu \Delta t\right)}, \\
& \gamma_{\mathrm{TS}}=\frac{\alpha_{0}^{2}+\alpha_{0} \alpha_{1}-\left(2 \alpha_{0} \beta_{0} \mu+\left(\alpha_{1} \beta_{0} M+\alpha_{0} \beta_{1}\right)-\gamma_{0}^{2} L\right) \Delta t+\left(\beta_{0}^{2}+\beta_{1} \beta_{0}\right) M \Delta t^{2}}{\alpha_{2}^{2}\left(1-2 \beta_{2} \mu \Delta t\right)},
\end{aligned}
$$

defined for values of $\Delta t$ such that $1-2 \beta_{2} \mu \Delta t \neq 0$ and being

$$
M=\sup _{t \in[0, T]} \mathbb{E}\left|f^{\prime}(X(t))\right|^{2} .
$$


Proof We consider two numerical solutions $\left\{X_{n}\right\}_{n}$ and $\left\{Y_{n}\right\}_{n}$ with $\mathbb{E}\left|X_{0}\right|^{2}<\infty$ and $\mathbb{E}\left|Y_{0}\right|^{2}<\infty$, computed by (10) with stepsize $\Delta t$. Lemma 1 leads to

$$
\begin{aligned}
\alpha_{2}^{2}\left(1-2 \beta_{2} \mu \Delta t\right)\left|X_{n+1}-Y_{n+1}\right|^{2} \leq & \mid-\alpha_{1}\left(X_{n}-Y_{n}\right)-\alpha_{0}\left(X_{n-1}-Y_{n-1}\right)+\beta_{1} \Delta t\left(f\left(X_{n}\right)-f\left(Y_{n}\right)\right) \\
& +\beta_{0} \Delta t\left(f\left(X_{n-1}\right)-f\left(Y_{n-1}\right)\right)+\gamma_{1}\left(g\left(X_{n}\right)-g\left(Y_{n}\right)\right) \Delta W_{n} \\
& +\left.\gamma_{0}\left(g\left(X_{n-1}\right)-g\left(Y_{n-1}\right)\right) \Delta W_{n-1}\right|^{2} .
\end{aligned}
$$

Squaring the right-hand side of this inequality, passing to expectations and applying assumptions (6) and (7) leads to

$$
\begin{aligned}
\alpha_{2}^{2}\left(1-2 \beta_{2} \mu \Delta t\right) \mathbb{E}\left|X_{n+1}-Y_{n+1}\right|^{2} \leq & \left(\alpha_{1}^{2}-2 \alpha_{1} \beta_{1} \mu \Delta t+\gamma_{1}^{2} L \Delta t\right) \mathbb{E}\left|X_{n}-Y_{n}\right|^{2} \\
& +\left(\alpha_{0}^{2}-2 \alpha_{0} \beta_{0} \mu \Delta t+\gamma_{0}^{2} L \Delta t\right) \mathbb{E}\left|X_{n-1}-Y_{n-1}\right|^{2} \\
& +\beta_{1}^{2} \Delta t^{2} \mathbb{E}\left|f\left(X_{n}\right)-f\left(Y_{n}\right)\right|^{2}+\beta_{0}^{2} \Delta t^{2} \mathbb{E}\left|f\left(X_{n-1}\right)-f\left(Y_{n-1}\right)\right|^{2} \\
& +2 \alpha_{0} \alpha_{1} \mathbb{E}\left|\left\langle X_{n}-Y_{n}, X_{n-1}-Y_{n-1}\right\rangle\right| \\
& -2 \alpha_{1} \beta_{0} \Delta t \mathbb{E}\left|\left\langle X_{n}-Y_{n}, f\left(X_{n-1}\right)-f\left(Y_{n-1}\right)\right\rangle\right| \\
& -2 \alpha_{0} \beta_{1} \Delta t \mathbb{E}\left|\left\langle X_{n-1}-Y_{n-1}, f\left(X_{n}\right)-f\left(Y_{n}\right)\right\rangle\right| \\
& +2 \beta_{0} \beta_{1} \Delta t^{2} \mathbb{E}\left|\left\langle f\left(X_{n}\right)-f\left(Y_{n}\right), f\left(X_{n-1}\right)-f\left(Y_{n-1}\right)\right\rangle\right| .
\end{aligned}
$$

Let us now provide estimates for the last six summands in (14):

- estimate of $\mathbb{E}\left|f\left(X_{n}\right)-f\left(Y_{n}\right)\right|^{2}$. Applying the mean value theorem to the function $f$ leads to

$$
\mathbb{E}\left|f\left(X_{n}\right)-f\left(Y_{n}\right)\right|^{2} \leq M \mathbb{E}\left|X_{n}-Y_{n}\right|^{2} ;
$$

- estimate of $\mathbb{E}\left|f\left(X_{n-1}\right)-f\left(Y_{n-1}\right)\right|^{2}$. Proceeding as above leads to

$$
\mathbb{E}\left|f\left(X_{n-1}\right)-f\left(Y_{n-1}\right)\right|^{2} \leq M \mathbb{E}\left|X_{n-1}-Y_{n-1}\right|^{2} ;
$$

- estimate of $2 \mathbb{E}\left|\left\langle X_{n}-Y_{n}, X_{n-1}-Y_{n-1}\right\rangle\right|$. We use the well-known inequality $2 a b \leq a^{2}+b^{2}$, for any $a, b \in \mathbb{R}$, leading to

$$
2 \mathbb{E}\left|\left\langle X_{n}-Y_{n}, X_{n-1}-Y_{n-1}\right\rangle\right| \leq \mathbb{E}\left|X_{n}-Y_{n}\right|^{2}+\mathbb{E}\left|X_{n-1}-Y_{n-1}\right|^{2} ;
$$

- estimate of $2 \mathbb{E}\left|\left\langle X_{n}-Y_{n}, f\left(X_{n-1}\right)-f\left(Y_{n-1}\right)\right\rangle\right|$. By Cauchy-Schwarz inequality together with the useful formula $2 a b \leq a^{2}+b^{2}$, we obtain

$$
2\left|\left\langle X_{n}-Y_{n}, f\left(X_{n-1}\right)-f\left(Y_{n-1}\right)\right\rangle\right| \leq\left|X_{n}-Y_{n}\right|^{2}+\left|f\left(X_{n-1}\right)-f\left(Y_{n-1}\right)\right|^{2} .
$$

Hence,

$$
2 \mathbb{E}\left|\left\langle X_{n}-Y_{n}, f\left(X_{n-1}\right)-f\left(Y_{n-1}\right)\right\rangle\right| \leq \mathbb{E}\left|X_{n}-Y_{n}\right|^{2}+M \mathbb{E}\left|X_{n-1}-Y_{n-1}\right|^{2} ;
$$

- estimate of $2 \mathbb{E}\left|\left\langle X_{n-1}-Y_{n-1}, f\left(X_{n}\right)-f\left(Y_{n}\right)\right\rangle\right|$. We proceed as above, obtaining

$$
2 \mathbb{E}\left|\left\langle X_{n-1}-Y_{n-1}, f\left(X_{n}\right)-f\left(Y_{n}\right)\right\rangle \leq \mathbb{E}\right| X_{n-1}-\left.Y_{n-1}\right|^{2}+M \mathbb{E}\left|X_{n}-Y_{n}\right|^{2} ;
$$

- estimate of $2 \mathbb{E}\left|\left\langle f\left(X_{n}\right)-f\left(Y_{n}\right), f\left(X_{n-1}\right)-f\left(Y_{n-1}\right)\right\rangle\right|$. We proceed as above, obtaining

$2 \mathbb{E}\left|\left\langle f\left(X_{n}\right)-f\left(Y_{n}\right), f\left(X_{n-1}\right)-f\left(Y_{n-1}\right),\right\rangle\right| \leq M\left(\mathbb{E}\left|X_{n}-Y_{n}\right|^{2}+\mathbb{E}\left|X_{n-1}-Y_{n-1}\right|^{2}\right)$. 
Matching (15)-(20) with (14) leads to the thesis.

This result first allows us to provide a complete analysis of the exponential meansquare properties of one-step methods. Indeed, the following result holds.

Theorem 3 Under conditions (6) and (7), any one-step stochastic method

$$
\alpha_{2} X_{n+1}+\alpha_{1} X_{n}=\Delta t\left(\beta_{2} f\left(X_{n+1}\right)+\beta_{1} f\left(X_{n}\right)\right)+\gamma_{1} g\left(X_{n}\right) \Delta W_{n} .
$$

satisfies the following exponential mean-square stability inequality

$$
\mathbb{E}\left|X_{n+1}-Y_{n+1}\right|^{2} \leq \mathbb{E}\left|X_{0}-Y_{0}\right|^{2} e^{v t_{n+1}},
$$

with

$$
v=\frac{1}{\Delta t} \log \beta_{\mathrm{OS}}
$$

being

$$
\beta_{\mathrm{OS}}=\frac{\alpha_{1}^{2}-\left(2 \alpha_{1} \beta_{1} \mu-\gamma_{1}^{2} L\right) \Delta t+\beta_{1}^{2} M \Delta t^{2}}{\alpha_{2}^{2}\left(1-2 \beta_{2} \mu \Delta t\right)},
$$

defined for values of $\Delta t$ such that $1-2 \beta_{2} \mu \Delta t \neq 0$.

Proof We apply the thesis of Theorem 2 to the family of one-step methods (21), obtaining that

$$
\mathbb{E}\left|X_{n+1}-Y_{n+1}\right|^{2} \leq \beta_{\mathrm{OS}} \mathbb{E}\left|X_{n}-Y_{n}\right|^{2},
$$

with $\beta_{\mathrm{OS}}$ defined as in (23) and, by recursion,

$$
\mathbb{E}\left|X_{n+1}-Y_{n+1}\right|^{2} \leq \beta_{\text {OS }}^{n+1} \mathbb{E}\left|X_{0}-Y_{0}\right|^{2} .
$$

The thesis immediately follows by assuming $v=\frac{1}{\Delta t} \log \beta_{\mathrm{OS}}$.

We now aim to provide an exponential mean-square stability inequality for the complete two-step case (10). For this purpose, we need to specify the one-step method we use in order to recover the missing starting values $X_{1}$ and $Y_{1}$. Once this aspect is clarified, the following result holds.

Theorem 4 Under conditions (6) and (7), any two-step stochastic method (10) satisfies the following exponential mean-square stability inequality over a single step

$$
\mathbb{E}\left|X_{n+1}-Y_{n+1}\right|^{2} \leq \mathbb{E}\left|X_{0}-Y_{0}\right|^{2} e^{\eta t_{n+1}},
$$

with

$$
\eta=\frac{1}{\Delta t} \log \left(\zeta_{n+1}\right),
$$

where $\zeta_{n+1}$ is recursively defined by the three-term formula

$$
\zeta_{n+1}=\beta_{\mathrm{TS}} \zeta_{n}+\gamma_{\mathrm{TS}} \zeta_{n-1},
$$

with $\zeta_{1}=\beta_{\mathrm{OS}}$ and $\zeta_{2}=\beta_{\mathrm{TS}} \beta_{\mathrm{OS}}+\gamma_{\mathrm{TS}}$. We recall $\beta_{\mathrm{TS}}$ and $\gamma_{\mathrm{TS}}$ are given in (12), while $\beta_{\mathrm{OS}}$ is given in (23) and characterizes the one-step method employed to compute the missing starting values $X_{1}$ and $Y_{1}$. 
Proof The thesis immediately follows by recursively applying the thesis of Theorem 2 , and applying the inequality

$$
\mathbb{E}\left|X_{1}-Y_{1}\right|^{2} \leq \beta_{\mathrm{OS}} \mathbb{E}\left|X_{0}-Y_{0}\right|^{2},
$$

for the first step.

\section{Contractivity of one-step methods}

We now employ the results in Section 2, especially Theorem 3, to study the exponential mean-square contractive behaviour of one-step methods (21). According to Definition 1, a one-step method is exponential mean-square contractive if $v<0$ in (22), i.e. if

$$
0<\beta_{\mathrm{OS}}<1
$$

in (23). Let us analyse this condition on the case by case basis.

\subsection{Stochastic trapezoidal rule}

We now aim to study the behaviour of the stochastic trapezoidal rule

$$
X_{i+1}=X_{i}+\frac{1}{2} \Delta t f\left(X_{i}\right)+\frac{1}{2} \Delta t f\left(X_{i+1}\right)+g\left(X_{i}\right) \Delta W_{i},
$$

when applied to an exponentially mean-square stable SDE (5) generating contractive solutions. For this method, the value of $\beta_{\mathrm{OS}}$ in (23) is given by

$$
\beta_{\mathrm{OS}}=\frac{4+4(\mu+L) \Delta t+M \Delta t^{2}}{4(1-\mu \Delta t)} .
$$

Let us now illustrate the above result with a numerical example, given by a scalar SDE (5) with

$$
f(x)=-4 x-x^{3}, \quad g(x)=x .
$$

In this case, (6) holds with $\mu=-4$ and (7) holds with $L=1$. Since $\alpha=2 \mu+L=$ $-7<0$, the problem exhibits an exponentially mean-square contractive behaviour, according to Definition 1. In order to compute the stepsize restriction corresponding to (26), we estimate the value of $M(13)$ as follows

$$
M \approx \tilde{M}=\max _{i=0,1, \ldots, N}\left\{\mathbb{E}\left|f^{\prime}\left(X_{n}^{\mathrm{EM}}\right)\right|, \mathbb{E}\left|f^{\prime}\left(Y_{n}^{\mathrm{EM}}\right)\right|\right\},
$$

where $X_{n}^{\mathrm{EM}}$ is the numerical solution computed by the Euler-Maruyama method

$$
X_{n}^{\mathrm{EM}}=X_{n-1}^{\mathrm{EM}}+\delta t f\left(X_{n-1}^{\mathrm{EM}}\right)+g\left(X_{n-1}^{\mathrm{EM}}\right) \Delta W_{n},
$$

with initial value $X_{0}$, stepsize $\delta t$, over $N+1$ grid points, while $Y_{n}^{\mathrm{EM}}$ is the numerical solution computed by the Euler-Maruyama method

$$
Y_{n}^{\mathrm{EM}}=Y_{n-1}^{\mathrm{EM}}+\delta t f\left(Y_{n-1}^{\mathrm{EM}}\right)+g\left(Y_{n-1}^{\mathrm{EM}}\right) \Delta W_{n},
$$


with initial value $Y_{0}$. The result of the computation of (29) over 1000 paths with $X_{0}=1, Y_{0}=2, N=2^{15}$ and $\delta t=10 / N$ gives $\widetilde{M}<25$. Hence, according to (26), the trapezoidal method (27) inherits the contractive behaviour of the problem if

$$
\Delta t<\frac{28}{25} \text {. }
$$

In order to provide a numerical evidence of the sharpness of this bound, we apply the trapezoidal method with $X_{0}=1$ and $Y_{0}=2$ and obtain the patterns reported in Fig. 1, where the pointwise mean-square of the error over 1000 paths is computed and depicted, in correspondence of $\Delta t=75 / 128$ and $\Delta t=75 / 64$. The method applied with $\Delta t=75 / 128$ shows the exponential decay of the mean-square error, while such a decay is totally lost for $\Delta t=75 / 64$, confirming the proved theoretical result.

\section{Contractivity of two-step methods}

We now move to analysing the contractivity of two-step methods (10), specializing the result in Theorem 4 to a selection of methods. According to Definition 1, a twostep method is exponential mean-square contractive if $\eta<0$ in (24), i.e. if, after performing $n$ steps,

$$
0<\zeta_{n}<1
$$

with $\zeta_{n}$ defined in (25). Let us analyse this condition on the case by case basis.

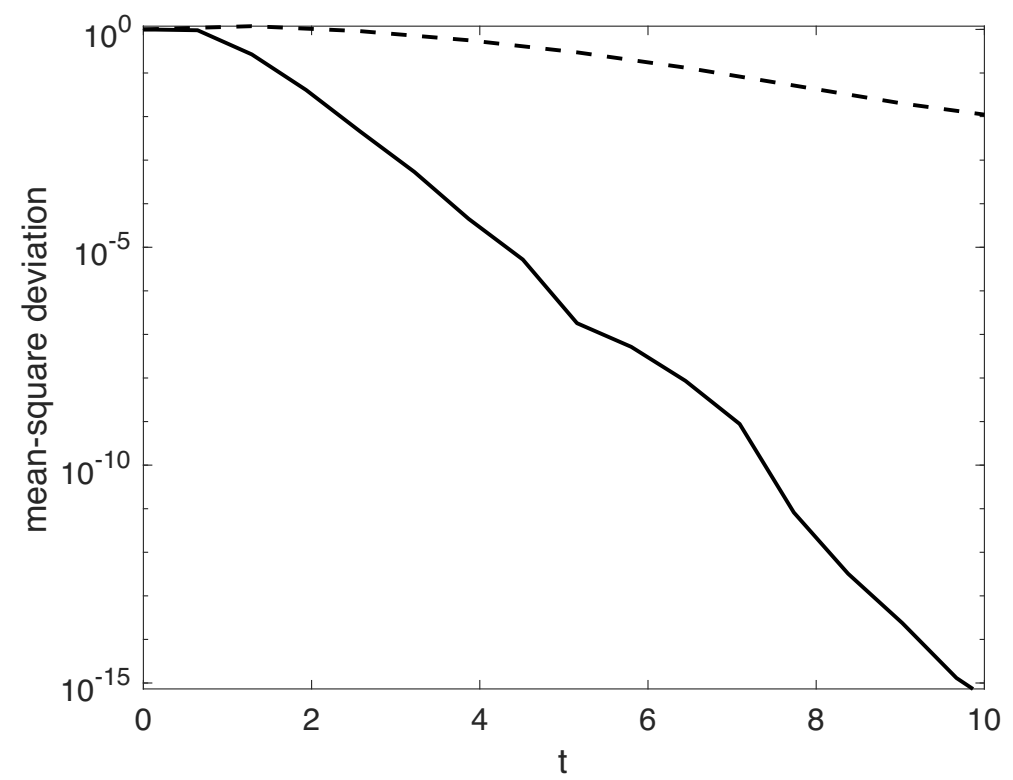

Fig. 1 Pattern of the mean-square deviation associated with the trapezoidal method applied to (5) with drift and diffusion given by (28), over 1000 paths with $X_{0}=0$ and $Y_{0}=1$, with stepsizes $\Delta t=75 / 128$ (solid line) and $\Delta t=75 / 64$ (dashed line) 


\subsection{Adams-Moulton method}

Adams-Moulton method is given by (10) with

$$
\begin{aligned}
& \alpha_{2}=1, \quad \alpha_{1}=-1, \quad \alpha_{0}=0, \beta_{2}=\frac{5}{12}, \\
& \beta_{1}=\frac{8}{12}, \beta_{0}=-\frac{1}{12}, \gamma_{1}=1, \quad \gamma_{0}=0 .
\end{aligned}
$$

For this method, we have

$$
\beta_{\mathrm{TS}}=\frac{36+(54 \mu-3+36 L) \Delta t+14 M \Delta t^{2}}{6(6-5 \mu \Delta t)}, \quad \gamma_{\mathrm{TS}}=\frac{M \Delta t}{24} \frac{7 \Delta t+12}{5 \mu \Delta t-6} .
$$

In order to check condition (30), we need to compute the values of $\zeta_{n}$ for large enough values of $n$, assuming to employ the trapezoidal method as a starting method. As a numerical illustration, we still consider problem (5) with drift and diffusion coefficients given by (28). For $n=30$, (30) leads to the following restriction on the stepsize

$$
\Delta t \leq 0.87
$$

in order to have exponential mean-square contractivity. The numerical evidence originated by applying the Adams-Moulton method with $X_{0}=1$ and $Y_{0}=2$ is reported in Fig. 2, where the pointwise mean-square of the error over 1000 paths is /computed and depicted, in correspondence of $\Delta t=25 / 64$ and $\Delta t=125 / 128$. The method applied with $\Delta t=25 / 64$ shows the exponential decay of the mean-square error, while such a decay is totally lost for $\Delta t=125 / 128$, confirming what we proved.

\subsection{Milne-Simpson}

Milne-Simpson method is given by (10) with

$$
\begin{aligned}
& \alpha_{2}=1, \quad \alpha_{1}=0, \quad \alpha_{0}=-1, \beta_{2}=\frac{1}{3}, \\
& \beta_{1}=\frac{4}{3}, \quad \beta_{0}=\frac{1}{3}, \quad \gamma_{1}=1, \quad \gamma_{0}=1 .
\end{aligned}
$$

For this method, we have

$$
\beta_{\mathrm{TS}}=\frac{3(4 M+3 L) \Delta t+20 M \Delta t^{2}}{3(3-2 \mu \Delta t)}, \quad \gamma_{\mathrm{TS}}=\frac{9+3(2 \mu+4+3 L) \Delta t+5 M \Delta t^{2}}{3(3-2 \mu \Delta t)} .
$$

In order to check condition (30), we need to compute the values of $\zeta_{n}$ for large enough values of $n$. By symbolic manipulation we have realized that, for large enough values of $n$, condition (30) is never fulfilled by positive values of $\Delta t$; thus, MilneSimpson method does not show an exponential mean-square stable character. 


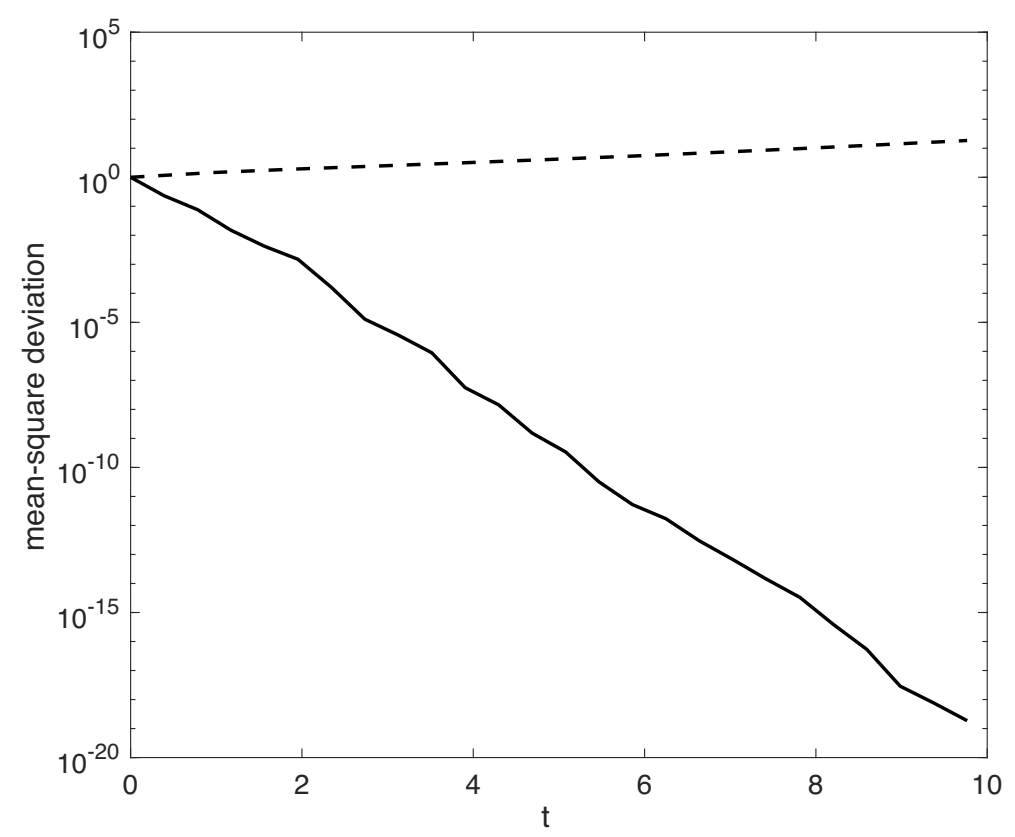

Fig. 2 Pattern of the mean-square deviation associated with the Adams-Moulton method applied to (5) with drift and diffusion given by (28), over 1000 paths with $X_{0}=0$ and $Y_{0}=1$, with stepsizes $\Delta t=$ $25 / 64$ (solid line) and $\Delta t=125 / 128$ (dashed line)

\subsection{BDF2}

BDF2 method is given by (10) with

$$
\begin{aligned}
& \alpha_{2}=1, \alpha_{1}=-\frac{4}{3}, \alpha_{0}=\frac{1}{3}, \quad \beta_{2}=\frac{2}{3}, \\
& \beta_{1}=0, \quad \beta_{0}=0, \quad \gamma_{1}=1, \quad \gamma_{0}=-\frac{1}{3} .
\end{aligned}
$$

For this method, we have

$$
\beta_{\mathrm{TS}}=\frac{3 L \Delta t+4}{3-4 \mu \Delta t}, \quad \gamma_{\mathrm{TS}}=\frac{L \Delta t-3}{3(3-4 \mu \Delta t)} .
$$

In order to check condition (30), we need to compute the values of $\zeta_{n}$ for large enough values of $n$, assuming to employ the trapezoidal method as a starting method. As a numerical illustration, we still consider problem (5) with drift and diffusion coefficients given by (28). For $n=30$, (30) does not lead to any stepsize restriction; in other terms, the method is nearly unconditionally stable for exponential mean-square contractivity. The numerical evidence originated by applying the Adams-Moulton method with $X_{0}=1$ and $Y_{0}=2$ is reported in Fig. 3, where the pointwise meansquare of the error over 1000 paths is computed and depicted, in correspondence of $\Delta t=25 / 64, \Delta t=25 / 32$ and $\Delta t=75 / 64$. Even for large values of the stepsize, 


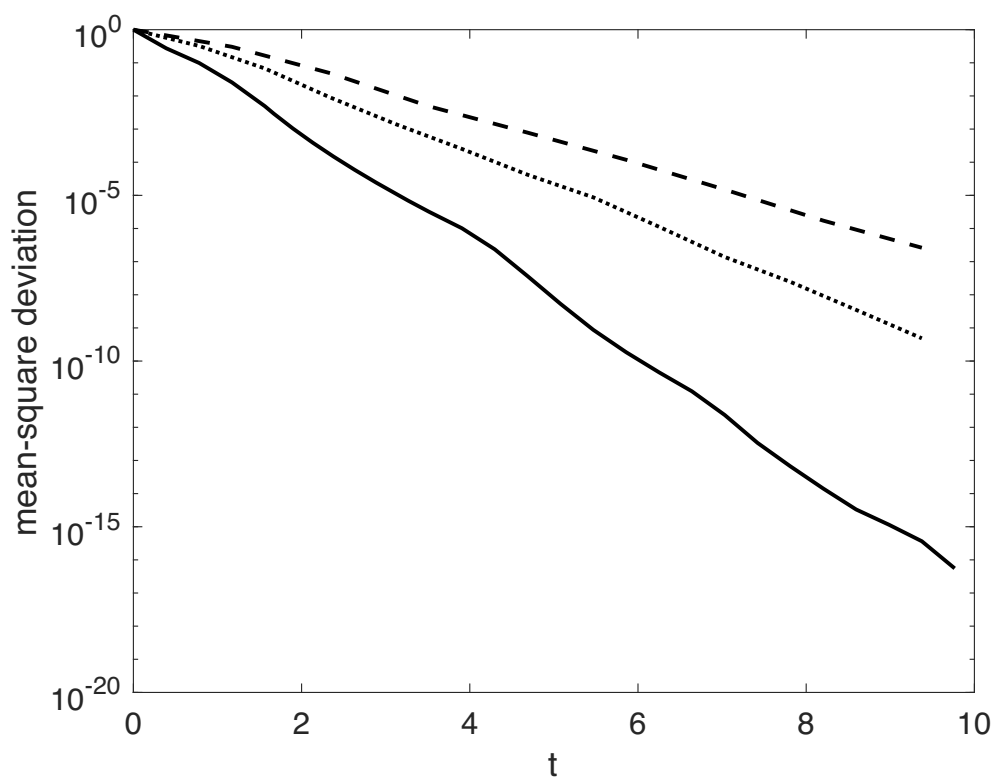

Fig. 3 Pattern of the mean-square deviation associated with the BDF2 method applied to (5) with drift and diffusion given by (28), over 1000 paths with $X_{0}=0$ and $Y_{0}=1$, with stepsizes $\Delta t=25 / 64$ (solid line), $\Delta t=25 / 32$ (dotted line) and $\Delta t=75 / 64$ (dashed line)

the exponential decay of the mean-square error is visible and, clearly, more accurate for smaller values of $\Delta t$.

\section{Numerical experiments}

As a test for the pertinence of the analysed methods in solving exponential meansquare stable SDEs (5), we provide the numerical evidence associated with the following nonlinear problems.

We first consider the following nonlinear SDE [16]

$$
d X(t)=-\frac{a}{1+t} X(t) d t+\frac{b}{1+t} X(t) \sin X(t) d W(t), \quad t \in[0,10],
$$

with $2 a-1 \geq b^{2}$ and $b \neq 0$ to ensure an exponential mean-square contractive behaviour, as proved in [16]. In our experiments, we choose $a=2$ and $b=1$. The evidence, reported in Fig. 4, shows the capability of trapezoidal, Adams-Moulton and BDF2 methods to preserve the expected mean-square contractivity.

We next consider the following nonlinear system [17]

$$
\left[\begin{array}{l}
d X_{1}(t) \\
d X_{2}(t)
\end{array}\right]=\left(A\left[\begin{array}{l}
X_{1}(t) \\
X_{2}(t)
\end{array}\right]+B\left[\begin{array}{c}
\sin X_{1}(t) \\
\cos X_{2}(t)
\end{array}\right]\right) d t+C\left[\begin{array}{l}
X_{1}(t) \\
X_{2}(t)
\end{array}\right]\left[\begin{array}{l}
d W_{1}(t) \\
d W_{2}(t)
\end{array}\right],
$$




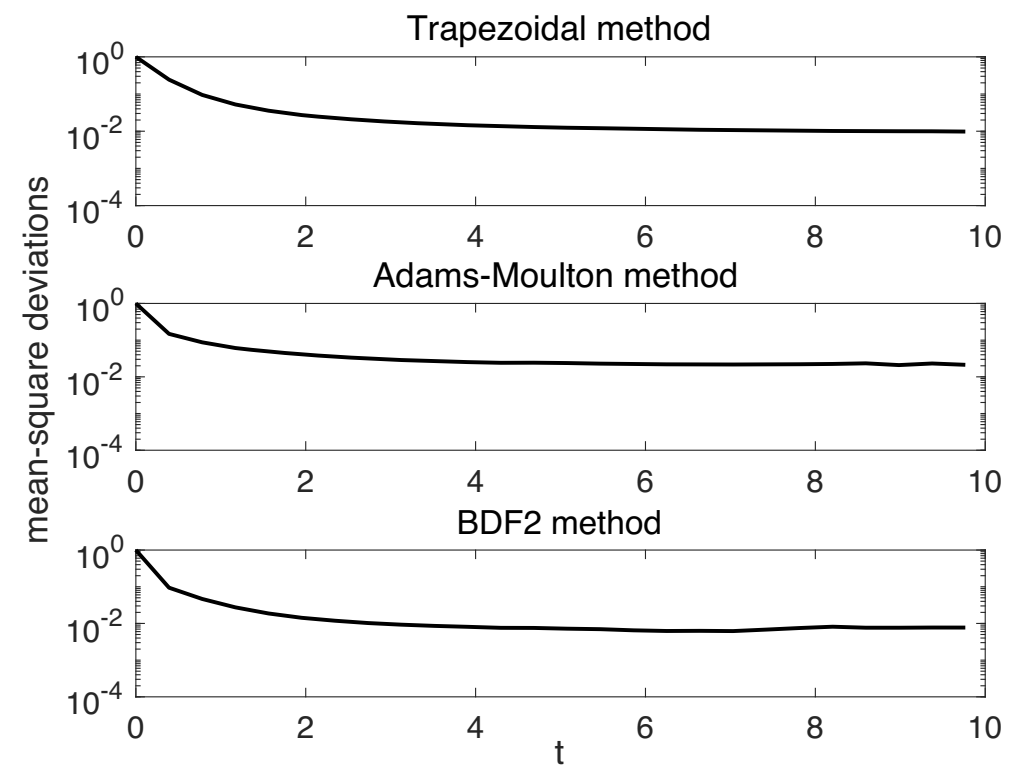

Fig. 4 Pattern of the mean-square deviations associated with the trapezoidal, Adams-Moulton and BDF2 methods applied to (31) over 1000 paths with $X_{0}=0$ and $Y_{0}=1$, with common stepsize $\Delta t=25 / 64$

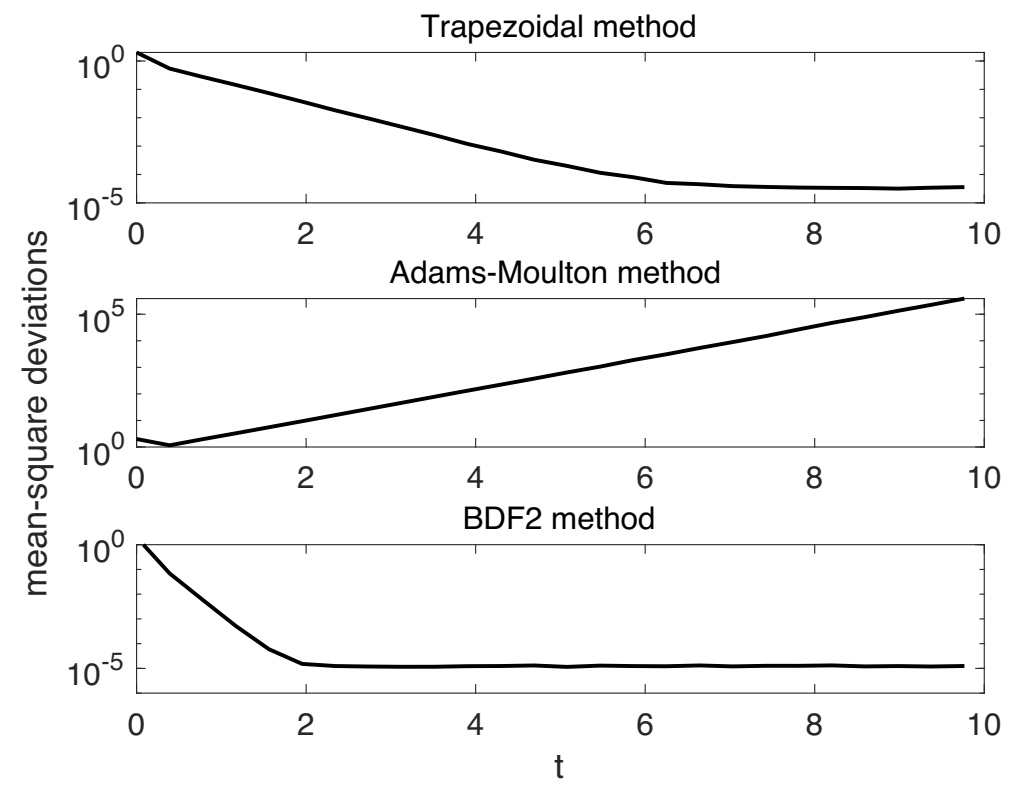

Fig. 5 Pattern of the mean-square deviations associated with the trapezoidal, Adams-Moulton and BDF2 methods applied to (32) over 1000 paths with $X_{0}=[0,1]^{\top}$ and $Y_{0}=[1,2]^{\top}$, with common stepsize $\Delta t=25 / 64$ 
where

$$
A=\left[\begin{array}{cc}
-28 & 0 \\
0 & -30
\end{array}\right], \quad B=\left[\begin{array}{cc}
2 & -1 / 2 \\
1 / 4 & 1
\end{array}\right], \quad C=\left[\begin{array}{cc}
1 & 3 / 2 \\
5 / 2 & -1 / 2
\end{array}\right],
$$

whose solution is exponentially mean-square stable (see [17]). Also in this case, the results reported in Fig. 5 show the capability of trapezoidal and BDF2 methods to nicely retain the mean-square contractivity behaviour, for $\Delta t=25 / 64$. AdamsMoulton method, subject to a more severe stepsize restriction, is not able to provide a mean-square contractivity behaviour for $\Delta t=25 / 64$.

\section{Conclusions}

We have analysed the behaviour of stochastic linear multistep methods when applied to nonlinear stochastic differential (5), in presence of exponential mean-square contractivity, under the conditions highlighted in Theorem 1. The theoretical analysis has provided sharp bounds on the stepsize to be employed in order to retain numerically the property of contractivity, as described in Sections 2-4. If a starting procedure is needed (and this is the case of two-step methods), the obtained bound also depends on the property of the employed starter method. Future issues of this research, which can be assumed as a starting point in assessing a theory of nonlinear stability for stochastic numerical methods, regard the possibility of extending the notion of algebraic stability for stochastic Runge-Kutta methods [8], in order to understand its possible connection with geometric numerical integration of stochastic Hamiltonian problems $[5]$.

Acknowledgements The second author is member of the GNCS-INDAM group. The authors are thankful to the anonymous reviewers for their precious comments.

Funding Open access funding provided by Università degli Studi dell'Aquila within the CRUICARE Agreement. This work is supported by GNCS-INDAM project and by PRIN2017-MIUR project 2017JYCLSF "Structure preserving approximation of evolutionary problems".

Open Access This article is licensed under a Creative Commons Attribution 4.0 International License, which permits use, sharing, adaptation, distribution and reproduction in any medium or format, as long as you give appropriate credit to the original author(s) and the source, provide a link to the Creative Commons licence, and indicate if changes were made. The images or other third party material in this article are included in the article's Creative Commons licence, unless indicated otherwise in a credit line to the material. If material is not included in the article's Creative Commons licence and your intended use is not permitted by statutory regulation or exceeds the permitted use, you will need to obtain permission directly from the copyright holder. To view a copy of this licence, visit http://creativecommons.org/licenses/by/4.0/.

\section{References}

1. Andersson, A., Kruse, R.: Mean-square convergence of the BDF2-Maruyama and backward Euler schemes for SDE satisfying a global monotonicity condition, arXiv:1509.00609 (2015)

2. Buckwar, E., Horvath-Bokor, R., Winkler, R.: Asymptotic mean-square stability of two-step methods for stochastic ordinary differential equations. Numer. Math. 101(1), 261-282 (2005) 
3. Butcher, J.C.: Thirty years of $G$-stability. BIT 46, 479-489 (2006)

4. Butcher, J.C. Numerical Methods for Ordinary Differential Equations, 2nd edn. John Wiley \& Sons, Chichester (2008)

5. Chen, C., Cohen, D., D'Ambrosio, R., Lang, A.: Drift-preserving numerical integrators for stochastic Hamiltonian systems. Adv Comput. Math. 46, article number 27 (2020)

6. Dahlquist, G.: A special stability property for linear multistep methods. BIT 3, 27-43 (1963)

7. Dahlquist, G.: Error analysis for a class of methods for stiff nonlinear initial value problems. In: Proc. Numer. Anal. Conf. (Dundee, Scotland, 1975), Lecture Notes Math., vol. 506, pp. 60-74. Springer, New York (1976)

8. D'Ambrosio, R., Di Giovacchino, S.: Nonlinear stability issues for stochastic Runge-Kutta methods, Comm. Nonlin. Sci. Numer. Simul. 94, article number 105549, https://doi.org/10.1016/j.cnsns.2020. 105549 (2021)

9. Dekker, K., Verwer, J.G.: Stability of Runge-Kutta Methods for Stiff Nonlinear Differential Equations CWI Monographs, vol. 2. North-Holland Publishing, Amsterdam (1984)

10. Emmrich, E.: Two-step BDF time discretisation of nonlinear evolution problems governed by monotone operators with strongly continuous perturbations. Comput. Methods Appl. Math. 9(1), 37-62 (2009)

11. Hairer, E., Wanner, G.: Solving Ordinary Differential Equations II - Stiff and Differential-Algebraic problems, 2nd Edition Springer (1996)

12. Higham, D.J., Kloeden, P.E.: Numerical methods for nonlinear stochastic differential equations with jumps. Numer. Math. 101(1), 101-119 (2005)

13. Higham, D.J., Mao, X., Stuart, A.M.: Strong convergence of Euler-type methods for nonlinear stochastic differential equations. SIAM J. Numer. Anal. 40(3), 1041-1063 (2002)

14. Higham, D.J., Mao, X., Stuart, A.M.: Exponential mean-square stability of numerical solutions to stochastic differential equations. LMS J. Comput. Math. 6, 297-313 (2003)

15. Higueras, I.: Strong stability for additive Runge-Kutta methods. SIAM J. Numer. Anal. 44(4), 17351758 (2006)

16. Mao, X.: Stability of stochastic differential equations with respect to semimartingales, Longman Scientific \& Technical, Pitman Research Notes in Mathematical Series 251, (1991)

17. Mao, X.: Exponential stability of stochastic differential equations. Marcel Dekker, Inc., New York (1994)

18. Tocino, A., Senosiain, M.J.: Asymptotic mean-square stability of two-step Maruyama schemes for stochastic differential equations. J. Comput. Appl. Math. 260, 337-348 (2014)

Publisher's note Springer Nature remains neutral with regard to jurisdictional claims in published maps and institutional affiliations. 